Documentation et bibliothèques

DOCUMENTATION BIBLIOTHËQUES

\title{
I - La publication académique face aux défis du marché et aux nouvelles technologies
}

\section{Brigitte Lévy}

Volume 49, numéro 4, octobre-décembre 2003

URI : https://id.erudit.org/iderudit/1030173ar

DOI : https://doi.org/10.7202/1030173ar

Aller au sommaire du numéro

Éditeur(s)

Association pour l'avancement des sciences et des techniques de la

documentation (ASTED)

ISSN

0315-2340 (imprimé)

2291-8949 (numérique)

Découvrir la revue

Citer ce document

Lévy, B. (2003). I - La publication académique face aux défis du marché et aux nouvelles technologies. Documentation et bibliothèques, 49(4), 177-180.

https://doi.org/10.7202/1030173ar

Tous droits réservés (C) Association pour l'avancement des sciences et des techniques de la documentation (ASTED), 2003
Ce document est protégé par la loi sur le droit d'auteur. L'utilisation des services d’Érudit (y compris la reproduction) est assujettie à sa politique d'utilisation que vous pouvez consulter en ligne.

https://apropos.erudit.org/fr/usagers/politique-dutilisation/ 


\title{
Les nouveaux modes de diffusion et l'accès aux publications savantes
}

\author{
N D L R
}

Les deux textes qui suivent ont été présentés à la Table ronde sur «Les nouveaux modes de diffusion et l'accès aux publications savantes", à l'occasion du $30^{\circ}$ Congrès annuel et colloque de l'ASTED tenu à Gatineau du 5 au 8 novembre 2003 . Les conférenciers y traitaient de la transformation du processus de la communication savante et de son impact sur les pratiques de recherche.

\section{I -La publication académique face aux défis du marché et aux nouvelles technologies}

\author{
Brigitte Lévy \\ Présidente, Association canadienne des revues savantes/ Canadian Association of Learned Journals \\ levy@management.uottawa.ca
}

es connaissances véhiculées par

L les revues savantes font partie du patrimoine culturel d'un pays et la communauté scientifique, dans son ensemble, bénéficie des résultats des recherches qui y sont publiés. À l'heure actuelle, le système de diffusion des résultats des recherches dans les sciences humaines et sociales, tout comme dans le domaine scientifique en général, fait face à plusieurs défis que je me propose d'aborder aujourd'hui.

\section{Les technologies de l'information et des communications (TIC): un nouveau paradigme technologique de diffusion de la recherche?}

L'émergence des TIC, dont le développement s'est considérablement accéléré au cours des deux dernières décennies, bouleverse les habitudes de diffusion de la recherche et de la publication. Nous sommes confrontés aux réalités de la société technologique et nous devons y faire face. Mais nous ne pourrons progresser sans nous interroger sur l'ampleur des changements, à savoir les enjeux économiques, sociaux, légaux, de nature académique et démocratique, qui accompagnent ce phénomène.

Je m'attarderai plus particulièrement à la publication académique des revues savantes. Selon un document publié par les Presses de l'Université de Montréal (1997), ces revues seraient en crise depuis plusieurs années. Une baisse des subventions, une augmentation du coût de l'impression et une diminution du nombre des abonnés figurent parmi les facteurs explicatifs de ce phénomène. De nouvelles modalités de publication, telle la publication en ligne, mériteraient donc d'être prises en considération.

On peut se demander si sous l'influence de la nouvelle économie et des innovations technologiques, la publication traditionnelle ne serait pas vouée à disparaître? D'emblée, ma réponse est négative, tout au moins à court et à moyen termes. Une période de transition est nécessaire où l'on pourra anticiper la juxtaposition des deux modes de publication. En ce qui concerne le long terme, seul l'avenir, à travers l'évolution de la société, des habitudes de consommation et l'ajus-' tement aux coûts de transition, sera en mesure de confirmer une tendance précise. Pour le moment, il serait prématuré de penser que l'édition et la diffusion électroniques se substitueront à l'ensemble de la publication imprimée des revues savantes.

Ceci ne veut pas dire que la publication électronique ne doit pas être envisagée comme une solution intéressante et viable. Elle peut permettre la diffusion rapide des idées et des documents. Certains observateurs avancent que la tech- nologie favorise l'épanouissement des principes démocratiques et permet de répondre aux demandes de la société du savoir. Pour d'autres, cependant, cette technologie engendre de nouvelles formes de leadership et de pouvoir.

Nous traversons indéniablement une période de transition industrielle qui nécessite une réorganisation continue de nos modes de production, de distribution et de concurrence. Les nouvelles technologies transforment la façon dont les entreprises élaborent, diffusent et soutiennent les produits traditionnels. Dans le cadre de l'édition savante, elles permettent d'élargir notre horizon et d'envisager de nouveaux modes de publication et de gestion. Des gains de productivité peuvent être enregistrés en raison de la rapidité de la communication entre les rédacteurs de revues, les auteurs, les évaluateurs et les autres intervenants impliqués dans le processus de production.

Mais, avant de faire l'apologie des nouvelles technologies et de vouloir les généraliser à l'ensemble des revues savantes, nous devons nous attarder à la question des coûts et des bénéfices sousjacents. Comme le demande Jody Berland (juin 2003), pouvons-nous réellement affirmer que les nouvelles technologies vont permettre de réduire à la fois les prix et les coûts à long terme? Est-ce que les technologies anciennes (imprimerie) coûtent cher en raison de leurs techniques de production et de diffusion à 
Si les universités, berceau de la recherche académique, consacrent de moins en moins de fonds aux revues, qui va se charger de prendre la relève et quels vont être les objectifs poursuivis?

proprement parler ou en raison tout simplement du système de publication à but non lucratif dans lequel elles opèrent?

\section{Les coûts et les bénéfices d'un nouveau mode de publication électronique}

Les années 90 ont été caractérisées par l'apparition progressive des revues électroniques et des «e-livres». La transition s'opère par à-coups. En ce qui concerne la mise en marché des livres, la recherche d'un appareil de lecture efficace et peu coûteux se poursuit. II s'agit là d'un type de publication qui connaîtra des débouchés dans des marchés spécifiques (stratégie de niche) où ils sont adaptés, mais, comme le mentionnent Hawkis et Forbes (2003), le livre électronique ne remplacera pas le livre imprimé. On peut aussi s'attendre à ce que la revue électronique soit un complément à la revue imprimée, plutôt qu'un substitut. Même s'il existe une volonté de la part des organismes subventionnaires ( $\mathrm{CRSH}$ ) d'encourager la publication des revues savantes en ligne, tous les rédacteurs de revues imprimées ne sont pas prêts à faire le grand saut.

L'application des nouvelles technologies aux processus de diffusion des revues savantes requiert d'importants investissements en matière de formation du personnel, d'achat de matériel et de logiciels. Un support informatique est nécessaire et ces technologies doivent être compatibles pour permettre une communication efficace entre tous les intervenants. En ce qui concerne la publication électronique, un accès ouvert à un grand nombre de participants est généralement souhaitable, mais elle soulève des questions en termes de perte potentielle de revenus d'abonnements. Pour les revues savantes, parmi les questions critiques figurent celles reliées aux coûts de production, aux revenus d'abonnements, à la formation du personnel, à un accès facile et bon marché aux outils de publication en ligne, à l'archivage et aux droits d'auteurs.

Notons aussi que, d'un point de vue qualitatif, l'évaluation par les pairs constitue l'élément central du processus de publication de la recherche académique. Les caractéristiques de l'évaluation par les pairs sur Internet doivent donc respecter l'intégralité et la légitimité de ce processus. L'évaluation par les pairs constitue une pratique fondamentale pour les rédacteurs des revues savantes et le monde académique dans son ensemble. Elle est le garant de la qualité d'une revue et la pierre angulaire de la publication académique. Pour les intervenants à ce processus, les questions d'avancement de carrière et d'évaluation de la recherche qui l'accompagnent, sont au cœur des pratiques de la profession. Nous ne devons, à aucun moment, perdre de vue ce rôle très important joué par les revues savantes en milieu académique. Il s'agit là d'une valeur dont la variable la plus importante n'est pas le prix mais la qualité intrinsèque du document publié.

À mon avis, à l'exception des nouvelles revues publiées d'emblée en ligne, la transition des revues savantes imprimées sous forme électronique sera longue et non uniforme. En règle générale, les revues savantes tirent avantage des nouveaux développements offerts par la technologie. Nombreuses sont celles qui possèdent un site Internet où sont publiées de nombreuses informations sur les numéros imprimés: politiques rédactionnelles, composition des comités de rédaction, noms des organismes de subventions, noms des auteurs, titres des articles et résumés de ces derniers. Les revues utilisent aussi le courriel pour communiquer rapidement avec les auteurs, les évaluateurs et les autres intervenants. Une mutation des processus managériaux sous l'impulsion des TIC est donc bel et bien en cours. Reste à évaluer l'ampleur des modifications induites par ces nouveaux outils de communication sur les processus de gestion des revues savantes. En particulier, il convient d'avoir une idée claire et précise des compétences requises pour les besoins de formation. Négliger de prendre en considération les aspects clés qui pourraient favoriser la transition vers la publication en ligne risque d'entraîner une réaction de résistance au changement. Nous devons nous interroger sur la perception des TIC et de la publication électronique par les revues savantes. Nous devons bien délimiter aussi les relations de pouvoir qui existent. Si les universités, berceau de la recherche académique, consacrent de moins en moins de fonds aux revues, qui va se charger de prendre la relève et quels vont être les objectifs poursuivis?

Des partenariats entre le secteur public et le secteur privé, c'est-à-dire un modèle de production dans lequel la notion de profit est présente, sont de plus en plus fréquents. De plus, les fonds de subventions fédéraux pour la recherche s'orientent beaucoup vers l'innovation technologique (Fondation canadienne pour l'innovation) et ne sont pas accessibles aux revues savantes. Pourtant, il existe une volonté de développer l'infrastructure nécessaire pour la publication en ligne. Ceci correspond à un choix politique et sociétal, répondant à divers objectifs. En particulier, les jeunes qui sont déjà initiés aux nouvelles technologies. Ils semblent posséder une culture moins "livresque " que leurs aînés et sont plus aptes à s'adapter aux changements de diffusion des connaissances induits par ces technologies. Cependant, envisager de mettre sur pied une infrastructure informatique de diffusion de la connaissance sans donner aux revues savantes les moyens financiers, techniques et humains pour qu'elles s'y adaptent progressivement pourrait s'avérer risqué.

Sans prendre en considération, pour le moment, le facteur coût, supposons que nous nous dirigions vers un système où cohabiteraient les revues imprimées et leurs versions en ligne, la question du contenu publié demeure préoccupante. 
Le contenu électronique est, de par sa nature, dynamique. Les versions PDF reproduisent les versions imprimées, mais les versions HTML peuvent enrichir le contenu, grâce à des liens qui fournissent des renseignements sur l'auteur, des références complémentaires, des citations et d'autres documents. Quelle est la frontière d'un article donné? Que doit-on archiver? De par sa nature, le contenu électronique permet une livraison efficace du matériel aux usagers et pourra peutêtre éventuellement diminuer les coûts de production. Cependant, la valeur réelle de la publication électronique demeure toujours inconnue.

La publication académique est un écosystème fragile (Ewing 2000). Les éditeurs des revues savantes valorisent la protection du savoir et des connaissances pour les générations présentes et futures. Les personnes qui participent à la publication savante et les rédacteurs de revues ont une responsabilité envers leur communauté et doivent être impliqués dans les processus décisionnels et les politiques qui initient le changement. Ces processus et politiques doivent tenir compte des caractéristiques propres aux publications savantes, dans le domaine des sciences sociales et humaines (mode de production à but non-lucratif; chaque revue opérant de façon autonome, avec ses caractéristiques propres).

\section{Bâtir le capital social de la publication électronique}

L'accès à Internet et à la publication en ligne procure une visibilité accrue pour les auteurs et théoriquement devrait permettre aux publications d'être diffusées à l'échelle mondiale. S'opposer à la publication électronique pourrait paraître antidémocratique et comme un refus de tenir compte des caractéristiques démographiques d'un segment de la population jeune et à l'aise avec la technologie (par rapport à l'ensemble de la génération qui la précède).

D'un point de vue sociétal, Internet permet ou devrait permettre la diffusion de la recherche à la communauté au sens large. Dans le cadre des revues savantes, un modèle est envisagé dans lequel les bibliothèques universitaires serviraient de plates-formes à la publication électronique de nombreuses revues, tout comme le font certains éditeurs commerciaux. Certains regroupements, tel celui proposé par «Synergies », qui s'inspire du modèle de publication «Érudit» de l'Université de Montréal, illustrent ce phénomène.

Pour l'édition savante en ligne, l'ère autodidacte a commencé au début des années 90 et se poursuit toujours. Selon Smith (2003), elle devient moins dominante en raison des coûts élevés du maintien et de l'amélioration de systèmes faits sur commande. Selon l'auteur, donner à une revue la possibilité d'acquérir des logiciels disponibles commercialement ou publiquement correspondant à ses besoins constituerait la prochaine étape. À titre d'exemple, le logiciel Open Journal System (OJS) de l'Université de la Colombie-Britannique permet l'affichage des articles sur le site Web, tout en gérant le processus de soumission, d'examen, d'acceptation, de révision, de correction, d'archivage et de préparation d'index Quand un auteur soumet un article, le système lui envoie automatiquement un courriel accusant réception.

La démarche est intéressante, mais nous devons être attentifs. Si l'article ne répond pas aux politiques rédactionnelles ou aux objectifs de la revue, le sys- d'index. Le système ne peut pas être téléchargé, mais les revues qui souhaitent l'utiliser pour se mettre en ligne peuvent l'obtenir «à un prix très raisonnable». Le projet a récemment mis au point un système appelé « my ICAAP», qui encourage davantage de personnes à participer au processus d'examen par les pairs. Et, je cite, «ce qui est significatif est de le faire non pas pour une revue mais plutôt pour leurs champs d'expertise». De nombreux évaluateurs seraient ainsi disponibles aux éditeurs de revues multiples. Qui va contrôler le processus d'évaluation par les pairs, l'élément qui donne la crédibilité aux revues et qui exerce une influence sur les décisions de carrières? En tout temps, des normes rigoureuses doivent être respectées (choix des évaluateurs les plus qualifiés, travail adéquat et respect des délais prescrits).

Un autre exemple de publication en ligne est le projet «Érudit» (Université de Montréal). Cependant, ce projet n'offre pas de services se rapportant à l'examen par les pairs ou aux aspects éditoriaux de l'édition. Le modèle suppose que les décisions de publication ou de révision ont déjà été prises et que la revue est prête à être publiée en ligne. Le modèle vise aus-

\section{S'opposer à la publication électronique pourrait paraître anti-démocratique et comme un refus de tenir compte des caractéristiques démographiques d'un segment de la population jeune et à l'aise avec la technologie.}

tème d'accusé de réception automatisé doit être en mesure de le faire savoir à l'auteur. Le même rapport intitulé L'édition savante en ligne au Canada: la technologie et les systèmes pour les sciences humaines et les lettres fait aussi état du projet de l'International Consortium for the Advancement of Academic Publishing (ICAAP - Consortium international pour l'avancement de l'édition académique), basé à l'Université d'Athabasca. II s'agit là d'un système qui utilise un modèle libre-service dans lequel tous les aspects de l'édition de la revue sont gérés, y compris l'examen par les pairs et l'ajout si la publication en ligne d'anciens numéros. Plusieurs sites canadiens, qui font partie du réseau Synergies, envisagent un tel système comme modèle de développement éventuel. Pour leur part, les Presses de l'Université de Calgary publient des livres et des revues et offrent à leur clientèle d'éditeurs, essentiellement en sciences sociales, un service complet allant de la gestion des manuscrits au développement de portails ainsi que leur appui. Je n'irai pas plus loin dans l'identification des projets en cours. Quelles que soient les limites ou les opportunités qu'ils présentent aux revues savantes, ces pro- 


\section{Si la publication académique électronique est postulée comme étant la voie du futur, on doit s'interroger sur ses contraintes, ses coûts, ses avantages et ses finalités.}

jets reflètent une tendance, non généralisée au sein de ces dernières, à s'orienter vers un nouveau mode de publication.

De par sa nature, la publication électronique des revues savantes traditionnelles va commander un modèle de fonctionnement plus intégré et peut-être même une vision stratégique globale. La problématique est déjà posée. En juin dernier, l'Association des bibliothèques de recherche du Canada a diffusé un document intitulé: Vers une stratégie de recherche canadienne pour la diffusion des connaissances. D'emblée, le ton est donné: « Les connaissances acquises dans le cadre des recherches savantes contribuent au bien-être économique, social et culturel d'un pays. Toutefois, ces recherches ont peu de valeur si elles ne sont pas partagées et diffusées à grande échelle. Actuellement, le système canadien de diffusion des connaissances scientifiques fait face à plusieurs défis, notamment: les nouvelles technologies". (L'étude en question bénéficie, entre autres, du financement du CRSH.)

Alors, doit-on en arriver à la conclusion que la publication électronique généralisée des revues savantes est un fait accompli dans l'esprit de certains organismes subventionnaires de la recherche et dans l'esprit aussi de certains rédacteurs de revues? La réponse est vraisemblablement positive. Mais le risque majeur consisterait à vouloir mettre la charrue avant les bœufs et à négliger un processus de consultation, d'information et de dialogue essentiel avec l'ensemble des responsables de la publication des revues savantes. Les rédacteurs de revues sont souvent préoccupés par la survie des processus de publication auxquels ils sont habitués - l'impression sur papier - et le nombre d'abonnés, de façon à couvrir une partie de leurs coûts de fonctionnement. La plupart des revues savantes opèrent avec des ressources financières et humaines limitées et n'ont pas les capacités techniques nécessaires pour se lancer dans la publication en ligne. Un modèle sociétal qui envisagerait le passage immédiat de la publication imprimée à la publication électronique de toutes les revuesn'est tout simplement pas réaliste.

Je suggère donc aux responsables des librairies universitaires, aux responsables académiques des universités et aux organismes subventionnaires de la recherche de s'interroger sur la valeur sociale des revues savantes au Canada. S'agit-il d'un bien public? Est-ce que le gouvernement et les institutions académiques qui parrainent les revues doivent continuer à jouer un rôle de support à la publication académique? Si la réponse est positive, nous devons nous préparer à en assumer les coûts, en donnant les moyens aux revues de continuer leur publication dans un format imprimé, tout en favorisant la publication électronique pour celles qui le désirent.

\section{Conclusion}

Tel que je perçois ce processus dans l'immédiat, la publication électronique ne réduira pas les coûts de fonctionnement des revues savantes traditionnelles, bien au contraire. Ce type de publication requiert la formation du personnel, l'accès aux techniques, des réseaux de distribution fiables et efficaces et un engagement ferme et volontaire de tous les intervenants. Pour être viable, toute stratégie de diffusion de la recherche doit créer un noyau cohérent de tous les participants au réseau. Si la publication académique électronique est postulée comme étant la voie du futur, on doit s'interroger sur ses contraintes, ses coûts, ses avantages et ses finalités.
Le thème du Congrès de l'ASTED est celui de L'ère des risques: savoir et choisir. Parmi les trois grandes conjonctures définissant cette ère, la troisième mentionnée: la recherche et la cohésion comme fondement du partenariat, s'applique bien à la problématique liée aux nouvelles mouvances de la diffusion de la recherche académique. Que la recherche porte sur les nouvelles lois relatives aux droits d'auteurs pour la publication électronique, sur les questions d'archivage des données ou sur le contenu savant des revues, un partenariat entre les principaux acteurs et la cohésion des processus décisionnels et de leurs modalités d'application sont nécessaires pour diminuer l'incertitude et le risque.

\section{Sources consultées}

Berland, Jody. June 2003. The Future of Academic Publishing. Presented to a Symposium on Academic Publishing with the Canadian Association of Research Libraries, The Canadian Association of Learned Journals and the Humanities and Social Sciences Federation of Canada, Halifax.

Ewing, John. 2003. Report on the NISO / NFAIS Workshop: Electronic Journals - Best Practices; Non-Profit Society Publisher. Philadelphia, PA, February (20).

Gellatly, Guy. 2003. L'économie canadienne en transition. Statistique Canada, Division microéconomique. Un guide pour les recherches sur la nouvelle économie, $\mathrm{n}^{0} 001$.

Hawkis, Donald T. et Forbes, Chris. 2003. The Pleasures and Pitfalls of Electronic Books, Info Today. (May 7).

Les Presses de l'Université de Montréal, Direction des publications électroniques. Un nouveau modèle de publication électronique. Site Internet www.pum.umontreal.ca/pum/publ_electr/vision.html, 17 décembre 1997.

Lévy, Brigitte. June 2003. Peer Reviewing. Presented to a Symposium on Academic Publishing with the Canadian Association of Research Libraries, The Canadian Association of Learned Journals and the Humanities and Social Sciences Federation of Canada, Halifax.

Smith, Richard. 2003. L'édition savante en ligne au Canada: la technologie et les systèmes pour les sciences humaines et les lettres. Advanced Publishing Research Lab, Simon Fraser University, Vancouver, BC. 